\section{MRSA Contamination of Computer Terminals}

Devine and colleagues from the District General Hospital, Eastbourne, East Sussex, United Kingdom, conducted a survey of two acute district general hospitals (A and $B$ ) to investigate the extent of methicillin-resistant Staphylococcus aureus (MRSA) contamination of wardbased computer terminals. Of 25 terminals examined, MRSA was identified in 6 (24\%). Environmental contamination was of a low level. Five of the MRSA-positive terminals were from hospital $\mathrm{A}$, which had a significantly higher rate of MRSA transmission compared to hospital B (1.02 vs 0.49 new inpatient MRSA cases per 100 hospital admissions for
1999). MRSA containment and handwashing policies were similar at both hospitals, but only hospital B actively audited handwashing compliance. It had a $44 \%$ higher rate of paper towel use per hospital bed.

Ward-based computer terminals pose a low risk of MRSA cross-infection. The authors believe that this risk can be further reduced if all staff wash their hands before and after patient contact

FROM: Devine J, Cooke RP, Wright EP. Is methicillinresistant Staphylococcus aureus (MRSA) contamination of ward-based computer terminals a surrogate marker for nosocomial MRSA transmission and handwashing compliance? J Hosp Infect 2001;48:72-75.

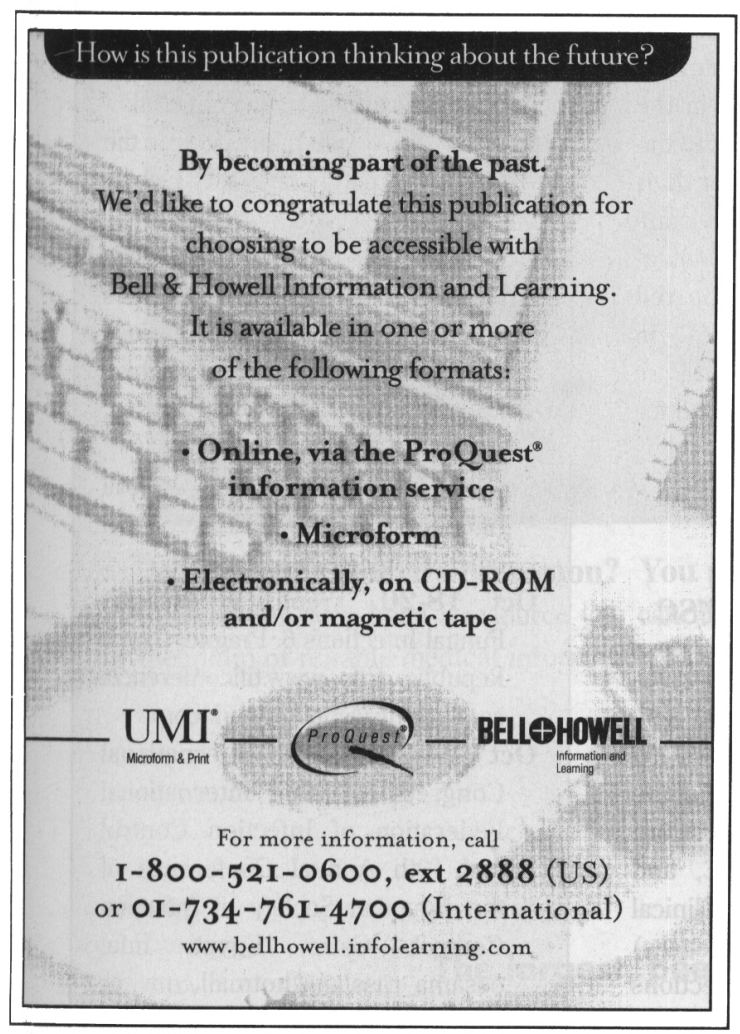

